\title{
Modifed pennes' equation modelling bio-heat transfer in living tissues: analytical and numerical analysis
}

\author{
Ahmed Lakhssassi ${ }^{1}$, Emmanuel Kengne $^{1^{*}}$, Hicham Semmaoui $^{2}$ \\ ${ }^{1}$ Laboratoire d'Ingénierie des Microsystèmes Avancés, Département d'informatique et d'ingénierie, Université du Québec en Ou- \\ taouais, Gatineau, Canada; 'Corresponding Author: kengem01@uqo.ca \\ ${ }^{2}$ Electronic Engineering Department, University of Montreal 2900 Chemin de la tour Montreal Qc H3C3A7, Montreal, Canada
}

Received 20 September 2010; revised 25 October 2010; accepted 28 October 2010.

\begin{abstract}
Based on modified version of the Pennes' bio-heat transfer equation, a simplified onedimensional bio-heat transfer model of the living tissues in the steady state has been applied on whole body heat transfer studies, and by using the Weierstrass' elliptic function, its corresponding analytic periodic and non-periodic solutions have been derived in this paper. Using the obtained analytic solutions, the effects of the thermal diffusivity, the temperature-independent perfusion component, and the temperature-dependent perfusion component in living tissues are analyzed numerically. The results show that the derived analytic solution is useful to easily and accurately study the thermal behavior of the biological system, and can be extended to applications such as parameter measurement, temperature field reconstruction and clinical treatment.
\end{abstract}

Keywords: Bio-Heat Transfer Equation; Pennes' Modified Equation; Weierstrass' Functions; Jacobi Elliptic Functions

\section{INTRODUCTION}

The effects of blood flow on heat transfer in living tissues have been examined for more than a century, dating back to the experimental studies of Bernard in 1876 [1]. Since that time, mathematical modelling of the complex thermal interaction between the vasculature and tissue has been a topic of interest for numerous physiologists, physicians, and engineers. It is a very difficult task to establish an appropriate physical model for the heat transport in the human body. The first quantitative relationship that described heat transfer in human tissue and included the effects of blood flow on tissue tem- perature on a continuum basis was presented by Harry $\mathrm{H}$. Pennes, a researcher at the College of Physicians and Surgeons of Columbia University [2]. His landmark paper, which appeared in 1948, is cited in nearly all research articles involving bio-heat transfer [3-10]. Appropriately, the equation derived in this paper is often referred to as the "traditional" or "classic" or "Pennes" bio-heat equation. The general form of Pennes' bio-heat equation is [7]

$$
\rho c \frac{\partial T}{\partial t}=\nabla(k \nabla T)+w_{b} c_{b}\left(T_{a}-T\right)+q_{m},
$$

where $\rho, c$, and $k$ are the density $\left(\mathrm{kg} / \mathrm{m}^{3}\right)$, the specific heat $(\mathrm{J} /(\mathrm{kg} . \mathrm{K}))$, and the tissue thermal conductivity $(W /(m . K))$, respectively; $w_{b}$ is the mass flow rate of blood per unit volume of tissue $\left(\mathrm{Kg} /\left(\mathrm{s} . \mathrm{m}^{3}\right)\right)$; $c_{b}$ is the blood specific heat; $q_{m}$ is the metabolic heat generation per unit volume $\left(\mathrm{W} / \mathrm{m}^{3}\right) ; T_{a}$ represents the temperature of arterial blood $(K) ; T$ is the temperature rise above the ambient level; $\partial T / \partial t$ is the rate of temperature rise. Here, we need to know the arterial blood temperature $T_{a}$. Pennes compressed all of the perfusion information into the term $w_{b} c_{b}\left(T-T_{a}\right)$. He checked the validity of this approximation by comparing temperatures predicted by his equation with experimentally measured temperatures in the human forearm. In his approach, the blood perfusion term $w_{b}$ was adjusted until the predicted temperatures agreed well with the measured temperatures. To facilitate the solution of the equation, the added blood perfusion term to account for perfusion heat transfer is linear in the temperature.

Pennes' primary premise was that energy exchange between blood vessels and the surrounding tissue occurs mainly across the wall of capillaries (blood vessels with $0.0054-0.015 \mathrm{~mm}$ in diameter) [7], where blood velocity is very low. He assumed, therefore, that the thermal contribution of blood can be modelled as if it enters an imaginary pool (the capillary bed) at the temperature of major supply vessels, $T_{a}$, and immediately equilibrates 
(thermally) with the surrounding tissue. Thus it exits the "pool" and enters the venous circulation at tissue temperature, $T$. He postulated therefore, that the total energy exchange by the flowing blood can be modelled as a nondirectional heat source, whose magnitude is proportional to the volumetric blood flow and the difference between local tissue and major supply arterial temperatures.

Boundary conditions are obtained from

$k \partial T / \partial n=h\left(T_{\text {out }}-T\right)$, where $T_{\text {out }}$ is the environmental temperature, and $h$ is the heat transfer coefficient. The main assumption of this model is that blood enters the local tissue with the body core temperature and leaves it with the local tissue temperature. Hence, convective heat flow can be described by the temperature difference $T-T_{b}$ and an empirical transfer coefficient $c_{b} w_{b}$ [11].

The mathematical model for heat transfer in tissue used to estimate tissue temperature profiles for each experiment based on tissue temperature measurements just before the animals were killed [12] was based on one-dimensional bio-heat equation that describes heat transfer in tissue [2] and was modified to account for temperature-dependent variability in tissue perfusion $[13,15]$. The model defines local tissue temperature $T(x, t)$ as

$$
\frac{\partial T}{\partial t}=\alpha \frac{\partial^{2} T}{\partial x^{2}}+\left[\omega_{0}+\omega_{1} \frac{T-T_{a}}{T_{a}}\right]\left(T_{a}-T\right),
$$

where $T$ is the local tissue temperature, $t$ is the time, $x$ is distance from the heated surface, $T_{a}$ is the input temperature of arterial blood, $\alpha$ is the thermal diffusivity, $\omega_{0}$ is the temperature-independent (basal) perfusion component, and $\omega_{1}$ is the temperature-dependent (vasodilation and angiogenesis) perfusion component. Eq.2 is a nonlinear version of the Pennes bio-heat transfer equation (temperature-dependent perfusion). Several simulations reveal that the temperature at the steady state is significantly higher with a temperature-dependent perfusion. Throughout this paper, Eq.2 will be referred to as the "modified" Pennes bio-heat (MPBH) equation.

Generally, the complexity of the nonlinear bio-heat transfer equations makes it difficult to obtain their analytic solutions. Many nonlinear equations can only be solved by numerical methods. However, analytic solutions of these equations, if attainable, are of important significance in the study of bio-heat transfer because they can accurately reflect not only the actual physical feature of equations but also be used as standards to verify the corresponding results of numerical calculation. In this paper, the derivation of analytic solution for onedimensional steady-state model of living tissue is conducted by adopting the applicable nonlinear bio-heat transfer equation. The rest of the paper is organized as follows. In section II, we present analytic solutions of the MPBH Eq.2 in the steady state. The effects of the thermal diffusivity, the temperature-independent perfusion component, and the temperature-dependent perfusion component are discussed in section III via numerical computation. The main results are summarized in section IV.

\section{ANALYTIC SOLUTIONS}

For analytic solutions, we suppose the input temperature $T_{a}$ of arterial blood to be constant. The steadystate condition is $\partial T / \partial t=0$ and the MPBH Eq. 2 becomes

$$
\frac{d^{2} T}{d x^{2}}=\frac{\omega_{1}}{\alpha T_{a}} T^{2}+\frac{\omega_{0}-2 \omega_{1}}{\alpha} T+\frac{T_{a}\left(\omega_{1}-\omega_{0}\right)}{\alpha} .
$$

Clearly, Eq.3 is a second-order nonlinear ordinary differential equation. If we multiply both sides of this equation by $d T / d x$ and integrate the resulting equation, we obtain

$$
\left(\frac{d T}{d x}\right)^{2}=4 \beta T^{3}+6 \gamma T^{2}+4 \delta T+\varepsilon=R(T),
$$

where

$$
\beta=\frac{\omega_{1}}{6 \alpha T_{a}}, \quad \gamma=\frac{\left(\omega_{0}-2 \omega_{1}\right)}{6 \alpha}, \quad \delta=\frac{\left(\omega_{1}-\omega_{0}\right) T_{a}}{2 \alpha},
$$

$\varepsilon$ being a constant of integration. The integration constant $\varepsilon$ plays the role of a control parameter. Eq.4 with the coefficients (5) is an elliptic ordinary differential equation and admits various periodic and solitary wave solutions, some of which can be found in Ref. [14,16].

As is well known $[17,18]$ the solution $T(x)$ of Eq.4 can be written as

$$
T(x)=T_{0}+\frac{R^{\prime}\left(T_{0}\right)}{4\left[\wp\left(x ; g_{2}, g_{3}\right)-\frac{1}{24} R^{\prime \prime}\left(T_{0}\right)\right]},
$$

where $T_{0}$ is a simple nonnegative root of $R(T)$ and the prime denotes differentiation with respect to $T$. The general solution $T(x)$ of Eq.4 is $[17,18]$

$$
T(x)=T_{0}+\frac{\sqrt{R\left(T_{0}\right)} \frac{d \wp\left(x ; g_{2}, g_{3}\right)}{d x}+\frac{R^{\prime}\left(T_{0}\right)}{2}\left[\wp\left(x ; g_{2}, g_{3}\right)-\frac{1}{24} R^{\prime \prime}\left(T_{0}\right)\right]+\frac{1}{24} R\left(T_{0}\right) R^{\prime \prime \prime}\left(T_{0}\right)}{2\left[\wp\left(x ; g_{2}, g_{3}\right)-\frac{1}{24} R^{\prime \prime}\left(T_{0}\right)\right]^{2}-\frac{1}{48} R\left(T_{0}\right) R^{\prime \prime \prime \prime}\left(T_{0}\right)},
$$


where $T_{0}$ is a real constant, not necessarily a zero of $R(T)$. The invariants $g_{2}$ and $g_{3}$ of Weierstrass' elliptic function $\wp\left(x ; g_{2}, g_{3}\right)$ are related to the coefficients of $R(T)$ by the relations [19]

$$
g_{2}=-4 \beta \delta+3 \gamma^{2}, g_{3}=2 \beta \gamma \delta-\gamma^{3}-\varepsilon \beta^{2} .
$$

The discriminant (of $\wp$ and $R$ [19])

$$
\Delta=g_{2}^{3}-27 g_{3}^{2},
$$

is suitable to classify the behavior of $T(x)$. The conditions

$$
\Delta \neq 0 \text { or } \Delta=0, g_{2}>0, g_{3}>0
$$

lead to periodic solutions [20], whereas the conditions [21]

$$
\Delta=0, g_{2} \geq 0, g_{3} \leq 0
$$

are associated with solitary wave-like solutions. We are interested in real, bounded, and nonnegative solutions $T(x)$. Based on form (6) for the solution $T(x)$, we list in what follows some interesting particular solutions of Eq.4 that cannot be found in Ref. [14].

\subsection{Case $\Delta>0$}

If $\Delta>0$, Weierstrass' function $\wp$ can be expressed as [21]

$$
\wp\left(x ; g_{2}, g_{3}\right)=e_{3}+\frac{e_{1}-e_{3}}{\operatorname{sn}^{2}\left(\sqrt{e_{1}-e_{3}} x, m\right)},
$$

where $s n(x, m)$ denotes the Jacobi elliptic sine function with modulus $m$ [14,22], $m=\left(e_{2}-e_{3}\right) /\left(e_{1}-e_{3}\right)$ and $e_{1} \geq e_{2} \geq e_{3}$ being the roots of the equation

$$
4 s^{3}-g_{2} s-g_{3}=0 .
$$

Substituting Eq.12 into Eq.6 yields [23] (14).

For the boundedness of solution (14) we must have either

$$
2 e_{3}-2 \beta T_{0}-\gamma \geq 0 \text { or } 2 e_{3}<2 \beta T_{0}+\gamma<2 e_{1} \text {. }
$$

It is easily seen that if conditions (15) are satisfied then $\left(2 e_{3}-2 \beta T_{0}-\gamma\right) \operatorname{sn}^{2}\left(\sqrt{e_{1}-e_{3}} x, m\right)+2\left(e_{1}-e_{3}\right)>0$ for every $x$, so that the bounded solution (14) will be nonnegative if, and only if, its numerator is nonnegative, i.e., if either (15).

Thus, Eqs.15 and 16 are boundedness and nonnegativity conditions, respectively, of solution (14). These are the mathematical conditions. For the clinical condition, we only need the boundedness and nonnegativity of $T(x)$ on some segment $\left[0, X_{0}\right]$. Indeed, if $X_{0}$ is the maximal distance from the heated surface, i.e., $x \leq X_{0}$ in Eq.2, and if $T(x)$ is nonnegative and bounded on the segment $\left[0, X_{0}\right]$, then we say that the clinical condition is valid.

Note: It should be noted that, in the case where $\Delta>0$, the polynomial $P(s)=4 s^{3}-g_{2} s-g_{3}$ cannot have a double root. Hence solution (14) contains neither a trigonometric nor a hyperbolic solution.

For the plot of this subsection we employed the thermal properties of tissue and blood based on Ref. [24]. The density of tissue is $1050 \mathrm{~kg} / \mathrm{m}^{3}$, both specific heat capacity of tissue and blood are $3770 \mathrm{~J} / \mathrm{kg}$. $\mathrm{C}$, and the thermal conductivity of tissue is $0.4 \mathrm{~W} / \mathrm{m} .{ }^{\circ} \mathrm{C}$. In addition, the arterial temperature is $37^{\circ} \mathrm{C}$. With these data, we compute $\alpha$ and $\omega_{0}$. The temperature-dependent perfusion component $\omega_{1}$ with be chosen properly.

Figure 1 shows the temperature profile along the distance from the heat source. For the curves of this figure, we used the periodic solution (14) with $\omega_{1}=6.449 \times$ $10^{-3} \mathrm{~s}^{-1}$. With this value of the temperature-dependent perfusion component, polynomial $R(T)$ admits three different roots: $T_{0}=38, T_{0}=35.634$, and $T_{0}=33.268$. These values of $T_{0}$ are used for plot (a), (b), and (c), respectively. Here, the free parameter $\varepsilon$ has been taken as $\varepsilon=\frac{\left(6 \tilde{a}^{2}-4 \tilde{a}^{3}-1\right) T_{a}^{2} \omega_{0}}{6 \alpha \tilde{a}^{2}}$, with $\tilde{a}=\frac{37(\sqrt{3}-1)}{2}$. As the plots of Figure 1 show, the temperature response is a steady periodic oscillation. The response temperature oscillates around the temperature $T_{0}$. We then set up the following question: It is true that the response temperature (14) will be always maintained almost at the input temperature of arterial blood $T_{a}^{\circ} \mathrm{C}$ if $T_{0}$ is a positive root of polynomial $R(T)$ so that $\left|T_{0}-T_{a}\right| \rightarrow 0$ ?

As we can see from Figure 2, the answer to the above question is negative! Figure $\mathbf{2}$ is obtained with the same parameters as in Figure 1, but with $\omega_{1}=\omega_{0}$ and $\varepsilon=456.33 \omega_{0} / \alpha$. With these values of parameters, we solved the equation $R(T)=0$ and as positive roots, we obtained $T_{0}=36.99^{\circ} \mathrm{C}$ and $T_{0}=37.058^{\circ} \mathrm{C}$. Plot (a) corresponds to $T_{0}=36.99^{\circ} \mathrm{C}$, while plot (b) is obtained using $T_{0}=37.058^{\circ} \mathrm{C}$. These two plots show that although $T_{0}$ is almost equal to the input temperature of

$$
\begin{gathered}
T(x)=\frac{\left(4 \beta T_{0}^{2}+2 e_{3} T_{0}+5 \gamma T_{0}+2 \delta\right) \operatorname{sn}^{2}\left(\sqrt{e_{1}-e_{3}} x, m\right)+2\left(e_{1}-e_{3}\right) T_{0}}{\left(2 e_{3}-2 \beta T_{0}-\gamma\right) \operatorname{sn}^{2}\left(\sqrt{e_{1}-e_{3}} x, m\right)+2\left(e_{1}-e_{3}\right)} . \\
4 \beta T_{0}^{2}+2 e_{3} T_{0}+5 \gamma T_{0}+2 \delta \geq 0 \text { and } T_{0} \geq 0 \text { or } 0<-\left(4 \beta T_{0}^{2}+2 e_{3} T_{0}+5 \gamma T_{0}+2 \delta\right)<2\left(e_{1}-e_{3}\right) T_{0} .
\end{gathered}
$$




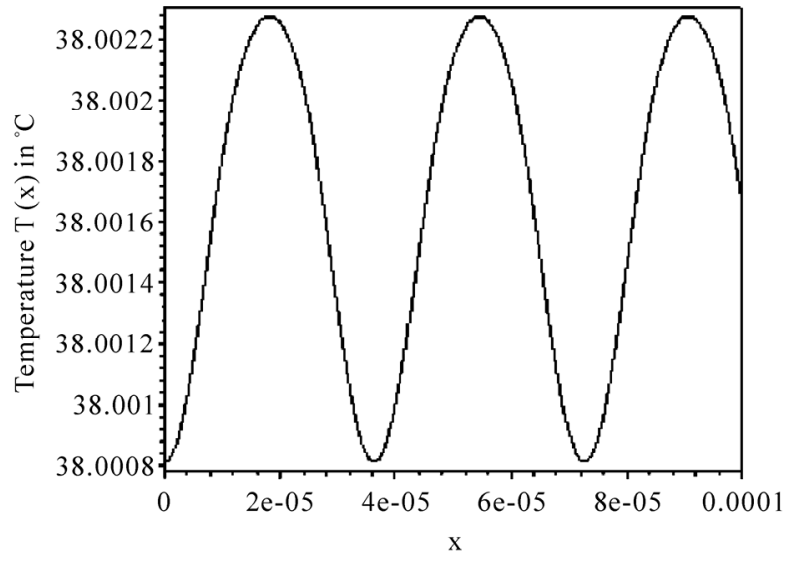

(a)

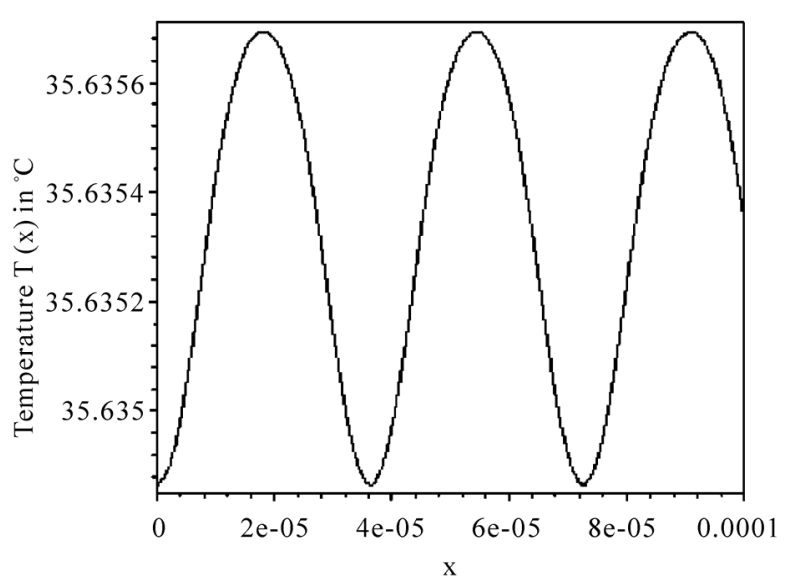

(b)

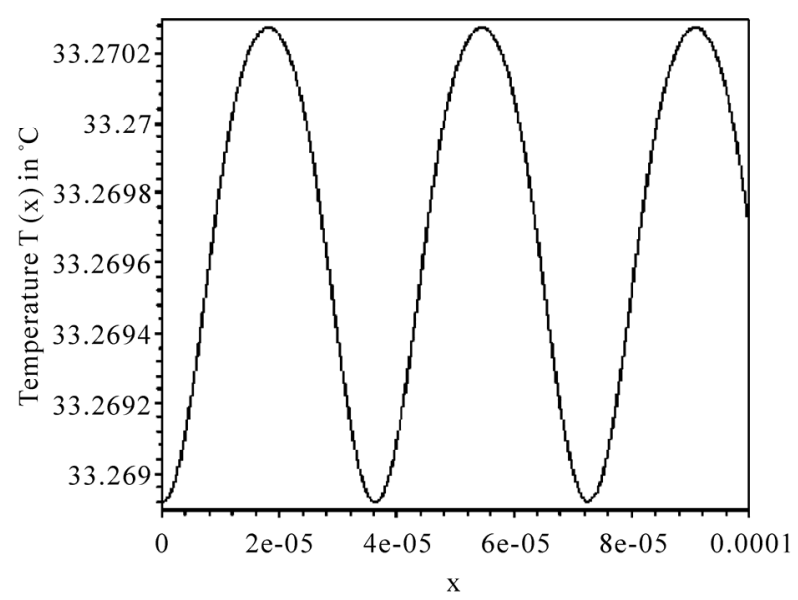

(c)

Figure 1. Temperature profiles along the distance $x$ (in $\mathrm{mm}$ ) from the heat source when $\alpha=1.2631 \times 10^{-7} \mathrm{~W} / \mathrm{J} / \mathrm{m}^{2}$, $\omega_{0}=4.7619 \times 10^{-4} \mathrm{~s}^{-1}, \quad T_{a}=37^{\circ} \mathrm{C}, \quad$ and $\omega_{1}=6.449 \times 10^{-3} \mathrm{~s}^{-1}$. The curves of this plot correspond to solution (14) with $T_{0}=38^{\circ} \mathrm{C} \quad$ (a), $T_{0}=35.63^{\circ} \mathrm{C}$ (b), and $T_{0}=33.268^{\circ} \mathrm{C}$ (c).

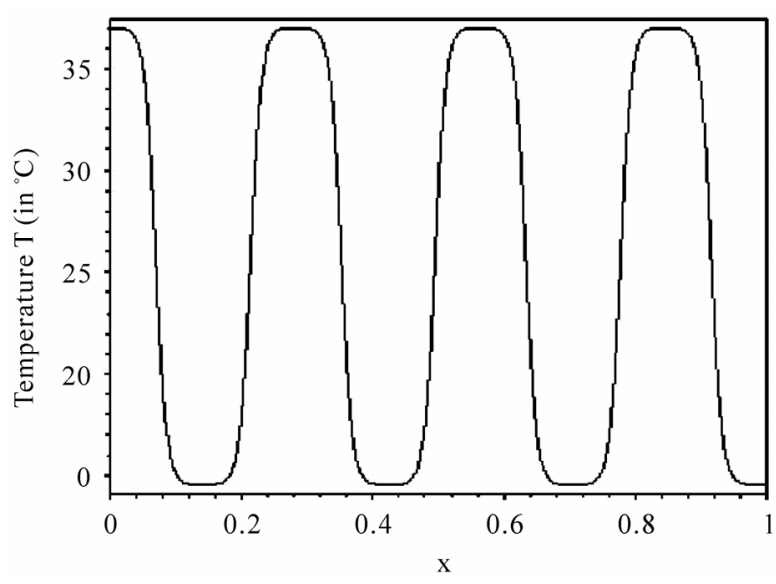

(a)

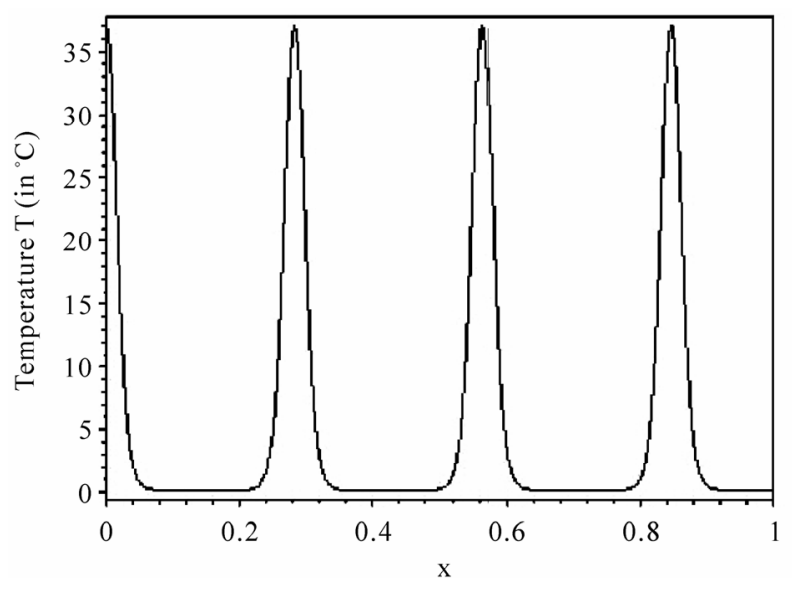

(b)

Figure 2. Temperature profiles along the distance $x$ (in $\mathrm{mm}$ ) from the heat source when using the same parameters as in Figure 1, but with $\omega_{1}=\omega_{0}$ and $\varepsilon=456.33 \omega_{0} / \alpha$. The curves of this plot correspond to solution (14) with $T_{0}=36.99^{\circ} \mathrm{C} \quad$ (a) and $T_{0}=37.058^{\circ} \mathrm{C} \quad$ (b).

arterial blood $T_{a}^{\circ} \mathrm{C}$, the response temperature corresponding to solution (14) oscillates far from $T_{a}$.

An obvious analysis of Eq.14 allows us to make the following conclusion: In order that the response temperature corresponding to the periodic solution (14) should be maintained at almost the input temperature of arterial blood $T_{a}^{\circ} \mathrm{C}$, it is necessary and sufficient that

$$
\begin{aligned}
& \left|T_{0}-T_{a}\right| \square 1 \text { and } \\
& 2\left(\delta+3 \gamma T_{0}+3 \beta T_{0}^{2}\right) /\left(2 e_{1}-2 \beta T_{0}-\gamma\right) \square 1 .
\end{aligned}
$$

For example, if we use the same parameters as in Fig. 1 but with $\omega_{1}=0.12898 \mathrm{~s}^{-1}$ and $\tilde{a}=270.86$, we compute the roots $T_{0}$ of $R(T)$ and find $T_{0}=37.05^{\circ} \mathrm{C}$, $T_{0}=36.932^{\circ} \mathrm{C}$, and $T_{0}=36.813^{\circ} \mathrm{C}$. Only one of these values of $T_{0}$, namely $T_{0}=36.813^{\circ} \mathrm{C}$, satisfies both conditions (15) and (16). With $T_{0}=36.813^{\circ} \mathrm{C}$ we have 


$$
\begin{aligned}
& \left|T_{0}-T_{a}\right|=|36.813-37|=0.187 \\
& \text { and } \frac{2\left(\delta+3 \gamma T_{0}+3 \beta T_{0}^{2}\right)}{2 e_{1}-2 \beta T_{0}-\gamma}=0.23838,
\end{aligned}
$$

and conditions (17) are satisfied. We then conclude that for $T_{0}=36.813^{\circ} \mathrm{C}$, the response temperature will be maintained at almost the input temperature of arterial blood $T_{a}^{\circ} \mathrm{C}$. This is confirmed by Figure 3.

$T_{0}=37.05^{\circ} \mathrm{C}$ and $T_{0}=36.932^{\circ} \mathrm{C}$ satisfy the clinical condition as one can see from Figure 3(a) and 3(c) where we used $T_{0}=37.05^{\circ} \mathrm{C}$ and $T_{0}=36.932^{\circ} \mathrm{C}$, respectively. For these $T_{0}=37.05^{\circ} \mathrm{C}$ and $T_{0}=36.932^{\circ} \mathrm{C}$ conditions (17) read

$$
\begin{aligned}
& \left|T_{0}-T_{a}\right|=|37.05-37|=0.05 \\
& \text { and } \frac{2\left(\delta+3 \gamma T_{0}+3 \beta T_{0}^{2}\right)}{2 e_{1}-2 \beta T_{0}-\gamma}=0.23503
\end{aligned}
$$

and

$$
\begin{aligned}
& \begin{array}{l}
\left|T_{0}-T_{a}\right|=|36.932-37|=0.068 \\
\text { and } \frac{2\left(\delta+3 \gamma T_{0}+3 \beta T_{0}^{2}\right)}{2 e_{1}-2 \beta T_{0}-\gamma}=46.462 .
\end{array} \\
& \begin{array}{l}
\left|T_{0}-T_{a}\right|=|36.932-37|=0.068 \\
\text { and } \frac{2\left(\delta+3 \gamma T_{0}+3 \beta T_{0}^{2}\right)}{2 e_{1}-2 \beta T_{0}-\gamma}=46.462 .
\end{array} \\
& T(x)=\frac{\left[\left(2 H-5 \gamma-2 e_{2}\right) T_{0}-4 \beta T_{0}^{2}-2 \delta\right] \operatorname{cn}(2 \sqrt{H} x, m)+2 \delta+4 \beta T_{0}^{2}+\left(2 H+5 \gamma+2 e_{2}\right) T_{0}}{\left(2 \beta T_{0}+2 H+\gamma-2 e_{2}\right) \operatorname{cn}(2 \sqrt{H} x, m)+2 H+2 e_{2}-2 \beta T_{0}-\gamma} . \\
& \left(2 H+2 e_{2}-2 \beta T_{0}-\gamma\right)\left(2 \beta T_{0}+2 H+\gamma-2 e_{2}\right)>0 \\
& \text { and } 2\left(2 e_{2}-\gamma-2 \beta T_{0}\right)\left(2 \beta T_{0}+2 H+\gamma-2 e_{2}\right)>0
\end{aligned}
$$

Thus, $T_{0}=37.05$ satisfies conditions (17), but $T_{0}=36.932$ does not satisfy this condition. Thus for $T_{0}=37.05$ the response temperature is maintained at almost the input temperature of arterial blood $T_{a}^{\circ} \mathrm{C}$ (see Figure 3(a)), while for $T_{0}=36.932$, the response temperature is not maintained at almost the input temperature of arterial blood $T_{a}^{\circ} \mathrm{C}$ (see Figure 3(c)). Figure 3(b) shows that condition $T_{0} \rightarrow T_{a}$ is not a sufficient condition for the response temperature to be maintained at almost the input temperature of arterial blood $T_{a}^{\circ} \mathrm{C}$.

\subsection{Case $\Delta<0$}

In the present case, we express Weierstrass' function $\wp$ as [21]

$$
\wp\left(x ; g_{2}, g_{3}\right)=e_{2}+H \frac{1+\operatorname{cn}(2 \sqrt{H} x, m)}{1-\operatorname{cn}(2 \sqrt{H} x, m)},
$$

where $m=1 / 2-3 e_{2} / 4 H, H^{2}=3 e_{2}^{2}+g_{2} / 4$, and $c n(x, m)$ is the Jacobi elliptic cosine function with modulus $m$. If we insert expression (18) into Eq.6 we obtain $\left(2 H+2 e_{2}-2 \beta T_{0}-\gamma\right)\left(2 \beta T_{0}+2 H+\gamma-2 e_{2}\right)<0$ and $4 H\left(2 \beta T_{0}+2 H+\gamma-2 e_{2}\right)<0$.

For the mathematical positivity of solution (19) it is necessary and sufficient that one of the following conditions should be satisfied:

1) Condition (20) is satisfied, moreover,

$$
\frac{2 \delta+4 \beta T_{0}^{2}+\left(2 H+5 \gamma+2 e_{2}\right) T_{0}}{\left(2 H-5 \gamma-2 e_{2}\right) T_{0}-4 \beta T_{0}^{2}-2 \delta}>0 \frac{4 \delta+8 \beta T_{0}^{2}+2 T_{0}\left(5 \gamma+2 e_{2}\right)}{\left(2 H-5 \gamma-2 e_{2}\right) T_{0}-4 \beta T_{0}^{2}-2 \delta}>0,
$$

and

$$
\frac{\left(2 H-5 \gamma-2 e_{2}\right) T_{0}-4 \beta T_{0}^{2}-2 \delta}{2 \beta T_{0}+2 H+\gamma-2 e_{2}}>0,
$$

2) Conditions (21) and (23) are satisfied and

$$
\frac{2 \delta+4 \beta T_{0}^{2}+\left(2 H+5 \gamma+2 e_{2}\right) T_{0}}{\left(2 H-5 \gamma-2 e_{2}\right) T_{0}-4 \beta T_{0}^{2}-2 \delta}<0 \frac{4 H T_{0}}{\left(2 H-5 \gamma-2 e_{2}\right) T_{0}-4 \beta T_{0}^{2}-2 \delta}<0,
$$

3) Condition (23) is violated when either conditions (20) and (24) are satisfied or conditions (21) and (22) are satisfied.

Figure 4 shows the plot of the response temperature along distance $x$ from the heated surface. Here we used the same parameters as in Figure 1, but with different values of the temperature-dependent perfusion component $\omega_{1}: \omega_{1}=7.119 \times 10^{-4} \mathrm{~s}^{-1}$ for plot (1), $\omega_{1}=6.5086 \times 10^{-4} \mathrm{~s}^{-1}$ for plot (2), and $\omega_{1}=6.3492 \times 10^{-4} \mathrm{~s}^{-1}$ for plot (3). For all these plots, we used $\varepsilon=0$ and 


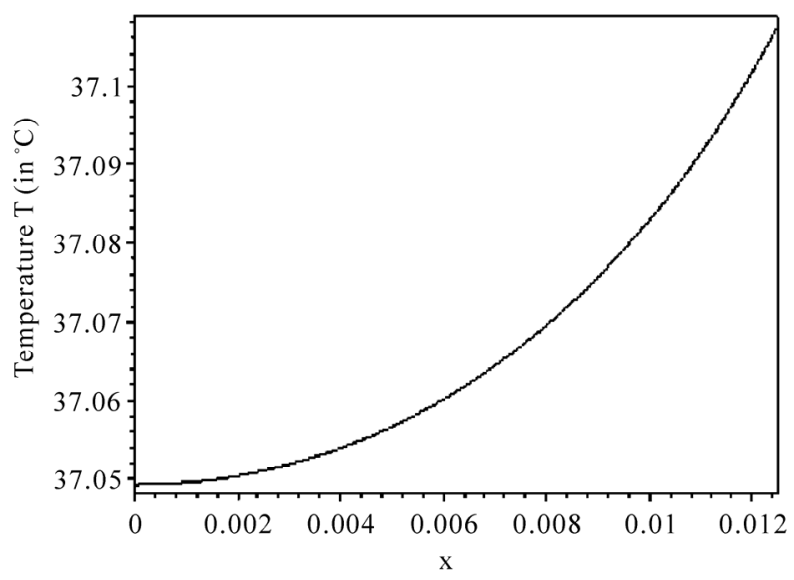

(a)

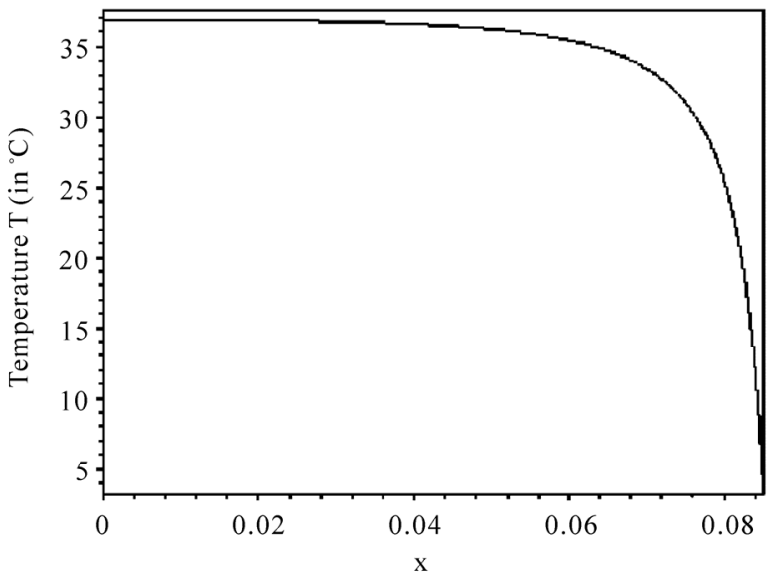

(b)

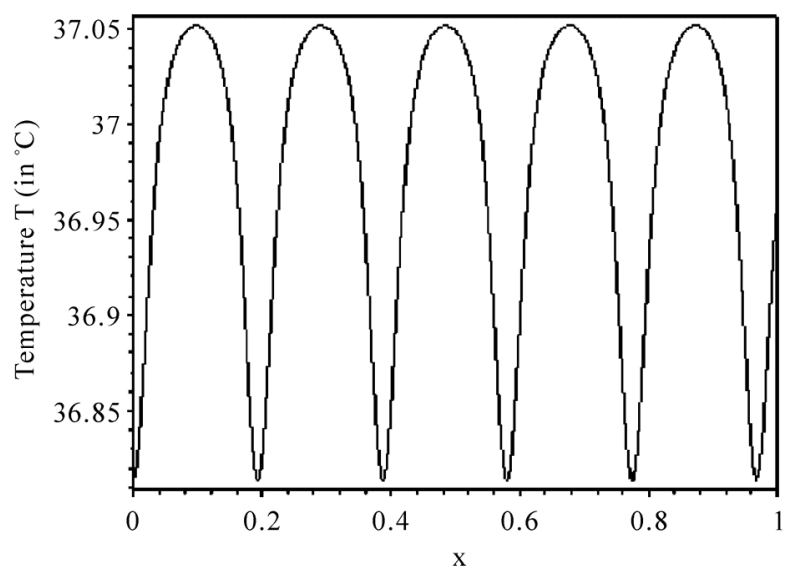

(c)

Figure 3. Plot of the response temperature along the distance $x$ (in $\mathrm{mm}$ ) from the heat source with the same parameter values as if Figure 1 but, with $\omega_{1}=0.12898 \mathrm{~s}^{-1}$ and $\tilde{a}=270.86$. The roots $T_{0}=37.05^{\circ} \mathrm{C}, T_{0}=36.932^{\circ} \mathrm{C}$, and $T_{0}=36.813^{\circ} \mathrm{C}$ have been used for curves (a), (b), and (c), respectively.

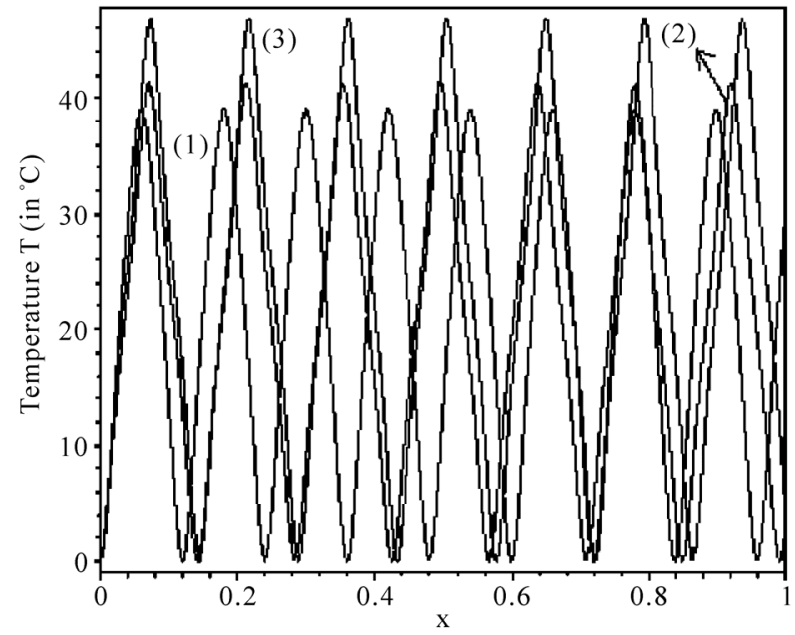

Figure 4. Plot of the response temperature along distance $x$ (in $\mathrm{mm}$ ) from the heated surface for the same parameter values as in Figure 1, but with different values of $\omega_{1}: \omega_{1}=7.119 \times 10^{-4} \mathrm{~s}^{-1}$ (1), $\omega_{1}=6.5086 \times 10^{-4} s^{-1}$ (2), and $\omega_{1}=6.3492 \times 10^{-4} s^{-1}$

$T_{0}=0$ in Eq.19. The curves of this figure show that for the same value of $T_{0}=0$, the maximal value of the response temperature increases as the temperature-dependent perfusion component $\omega_{1}$ decreases.

\subsection{Case $\Delta=0$}

Solving equation $\Delta=0$ with respect to $\varepsilon$, we obtain

$$
\varepsilon=-\left(\frac{\omega_{0}^{3}}{108 \alpha^{3}}+\frac{\omega_{1}^{3}}{54 \alpha^{3}}-\frac{\omega_{1}^{2} \omega_{0}}{36 \alpha^{3}}\right) \frac{36 \alpha^{2} T_{a}^{2}}{\omega_{1}^{2}} .
$$

Inserting this expression for $\varepsilon$ in the expression for $g_{3}$, we find that

$$
g_{3}=\frac{\omega_{0}^{3}}{213 \alpha^{3}},
$$

which is always positive. Hence in the case where $\Delta=0$, Weierstrass' function $\wp\left(x ; g_{2}, g_{3}\right)$ can be expressed by trigonometric functions [21] and (6) reads

$$
T(x)=T_{0}+\frac{R^{\prime}\left(T_{0}\right) \sin ^{2} \sqrt{\frac{3}{2} e_{1} x}}{12 e_{1}-\frac{24 e_{1}+R^{\prime \prime}\left(T_{0}\right)}{6} \sin ^{2} \sqrt{\frac{3}{2} e_{1} x}} \text {, if } \omega_{0} \neq 0 \text {, }
$$

and

$$
T(x)=T_{0}+\frac{6 R^{\prime}\left(T_{0}\right)}{24-R^{\prime \prime}\left(T_{0}\right) x^{2}} \text {, if } \omega_{0}=0 \text {, and } T_{0}<T_{a},
$$

where $e_{1}=\sqrt[3]{g_{3}} / 2$ in Eq.25, and $T_{0}$ is a simple root of $R(T)$. 
In Figure 5 we plot the response temperature $T(x)$ along the distance from the heated surface for the same parameters as in Figure 1 but with different values of $\omega_{1}: \omega_{1}=1.7619 \times 10^{-2} \mathrm{~s}^{-1}$ for plot (a), $\omega_{1}=4.7619 \times 10^{-5}$ $\mathrm{s}^{-1}$ for plot (b), and $\omega_{1}=1.6668 \times 10^{-3} \mathrm{~s}^{-1}$ for plot (c). For plots (a), (b), and (c) we used $T_{0}=37.7^{\circ} \mathrm{C}$, $T_{0}=38.85^{\circ} \mathrm{C}$, and $T_{0}=36.5^{\circ} \mathrm{C}$, respectively. Plots (a) and (b) correspond to solution (25), while plot (c) corresponds to solution (26).

\section{NUMERICAL COMPUTATION}

For the numerical computation of the nonlinear heat transfer Eq.2 we use the the Crank-Nicolson method, as it is stable and accurate to second order in space and time [25]. The accuracy of our numerical computations is checked by testing different time and space steps. The variables $t$ and $x$ are measured in units of time and space, respectively. In this study, we consider the input temperature $T_{a}$ of arterial blood as an $x$-dependent quantity. This input temperature is chosen among the steady-state solutions obtained in the previous section. Any other coefficient in Eq.2 is considered to be constant. We consider that the initial time is $t_{0}=0$ and distance $x$ from the heated surface is comprised between 0 and $X_{0}$ so that the smallest $x$ distance is $x_{0}=0$; furthermore we designate by $\tau$ and $h$ the time step and the $x$ step, respectively, so that $t$ will be incremented by $\tau$ and $x$ by $h$. We denote by $T_{j}^{n}$ the numerical value of the temperature at point $\left(x_{j}, t_{n}\right): T_{j}^{n} \approx T\left(x_{j}, t_{n}\right)$, where $t_{n}=n \tau$ and $x_{n}=j h$. Using $T_{a}(x)$ as the input temperature, at $t=0, T(x, 0)$ is set the same as $T_{a}(x)$. According to the Crank-Nicolson method, we use

$$
\begin{aligned}
& \frac{\partial T}{\partial t}=\frac{T_{j}^{n+1}-T_{j}^{n}}{\tau} ; \\
& \frac{\partial^{2} T}{\partial x^{2}}=\frac{1}{2}\left[\frac{T_{j+1}^{n}-2 T_{j}^{n}+T_{j-1}^{n}}{h^{2}}+\frac{T_{j+1}^{n+1}-2 T_{j}^{n+1}+T_{j-1}^{n+1}}{h^{2}}\right] .
\end{aligned}
$$

Inserting Eq.27 into Eq.2, we obtain

$$
\begin{aligned}
& -\frac{\alpha}{2 h^{2}} T_{j-1}^{n+1}+\left(\frac{1}{\tau}+\frac{\alpha}{h^{2}}\right) T_{j}^{n+1}-\frac{\alpha}{2 h^{2}} T_{j+1}^{n+1}=\frac{\alpha}{2 h^{2}} T_{j-1}^{n} \\
& +\left[\frac{1}{\tau}-\frac{\alpha}{h^{2}}+\left(2 \omega_{1}-\omega_{0}\right)-\frac{\omega_{1}}{T_{a}} T_{j}^{n}\right] T_{j}^{n} \\
& +\frac{\alpha}{2 h^{2}} T_{j+1}^{n}+T_{a}\left(\omega_{0}-\omega_{1}\right) .
\end{aligned}
$$

As $x$-dependent input temperature of arterial blood, we used either

$$
T_{a}(x)=\frac{-327.41 \mathrm{sn}^{2}\left(0.92823 \times 10^{5} x, 0.5\right)+654.83}{-8.6162 \mathrm{sn}^{2}\left(0.92823 \times 10^{5} x, 0.5\right)+17.232},
$$

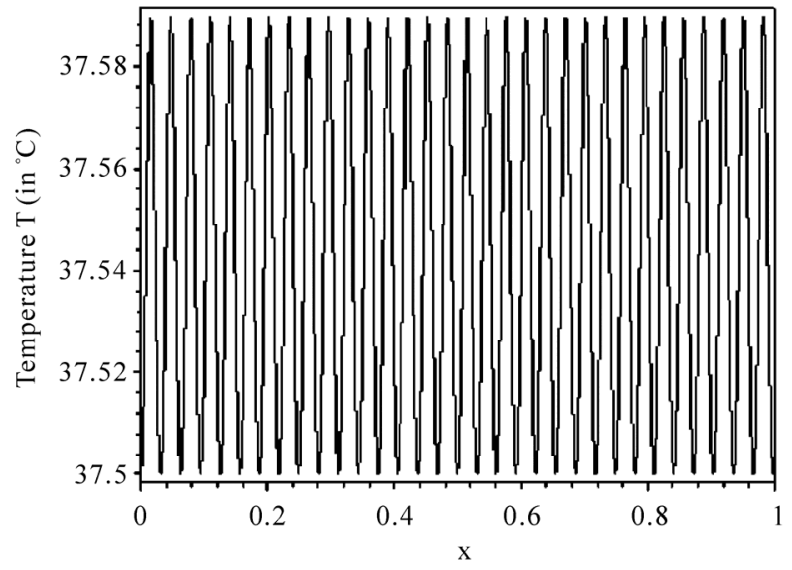

(a)

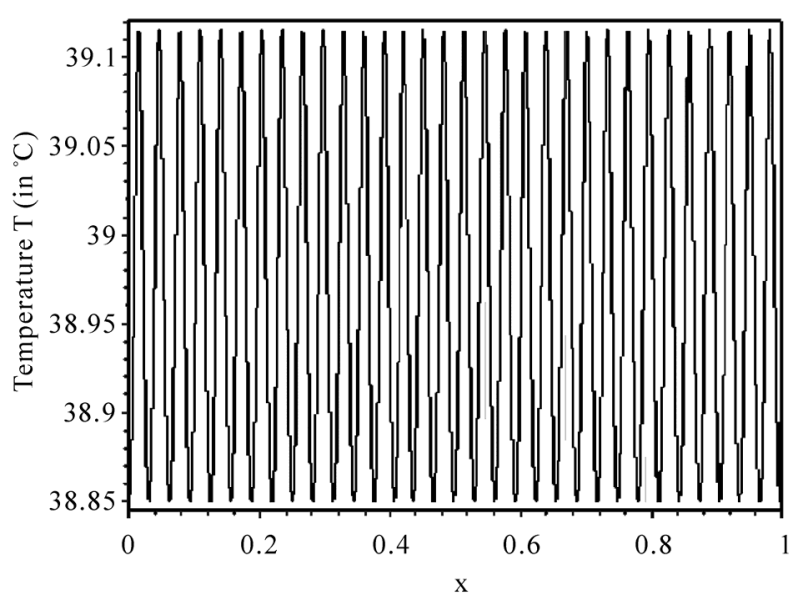

(b)

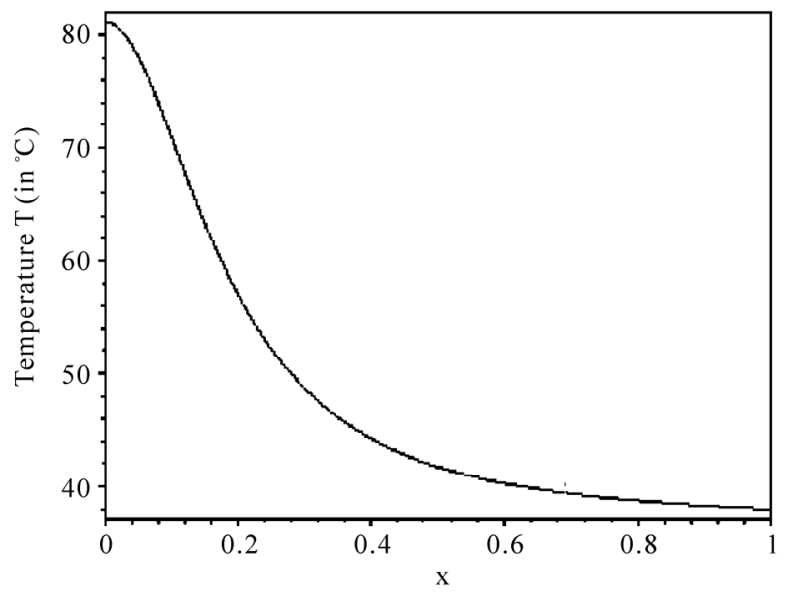

(c)

Figure 5. Plot of the response temperature along distance $x$ (in $\mathrm{mm}$ ) from the heated surface for the same parameter values as in Figure 1, but with different values of $\omega_{1}: \omega_{1}=1.7619 \times 10^{-2} \mathrm{~s}^{-1}$ (a), $\omega_{1}=4.76196 \times 10^{-5} \mathrm{~s}^{-1}$ (b), and $\omega_{1}=1.66686 \times 10^{-3} s^{-1} \quad$ (c). 
obtained from Eq.14, or

$$
T(x)=36.5+\frac{1070}{24+713.3 x^{2}},
$$

obtained from Eq.26. For the numerical simulation, we used Mathematica.

\subsection{Effect of the Thermal Diffusivity $\alpha$}

For the numerical study of the effect of thermal diffusivity, the following temperature-independent perfusion component and temperature-dependent perfusion component are used: $\omega_{0}=4.7619 \times 10^{-4} \mathrm{~s}^{-1}$ and

$\omega_{1}=6.449 \times 10^{-3} \mathrm{~s}^{-1}$, respectively. These parameters, together with $\alpha=1.2631 \mathrm{~W} / \mathrm{J} . \mathrm{m}^{2}$ have been used for plot (a) of Figure 1.

Figure 6 shows the numerical solution for different values of $\alpha$. The first, second, and third rows of this figure correspond to $\alpha=1.26311 \times 10^{-8} \mathrm{~W} / \mathrm{J} . \mathrm{m}^{2}$, $\alpha=1.26311 \times 10^{-4} \mathrm{~W} / \mathrm{J} . \mathrm{m}^{2}$, and $\alpha=1.26311 \times 10^{-2} \mathrm{~W} / \mathrm{J} . \mathrm{m}^{2}$, respectively. The plots of the first, second, and third columns show the temperature at time $t=50 \mathrm{~s}, t=80$ $\mathrm{s}$, and $t=100 \mathrm{~s}$, respectively. An $x$-dependent input temperature of arterial blood (28) is used. The plots of Figure 6 show that the response temperature decreases as the thermal diffusivity $\alpha$ increases.

\subsection{Effect of the Temperature-Independent Perfusion Component $\omega_{0}$}

To show the effect of the temperature-independent perfusion component $\omega_{0}$, we plotted Figures 7(a-f) with the parameter values $\alpha=1.26311 \times 10^{-7} \mathrm{~W} / \mathrm{J}^{\mathrm{m}} \mathrm{m}^{2}$ and

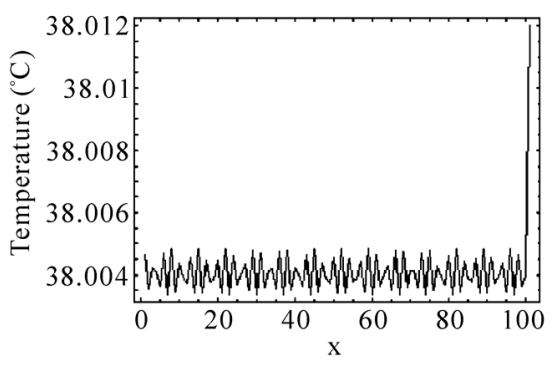

(a)

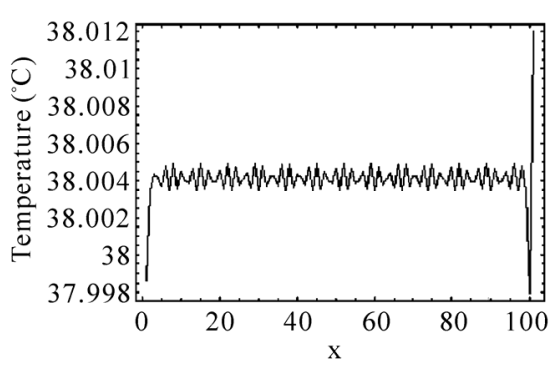

(d)

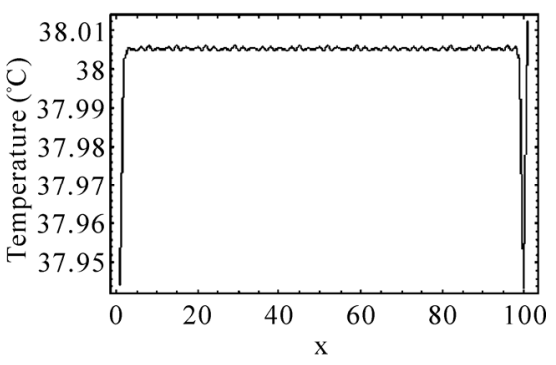

(g)

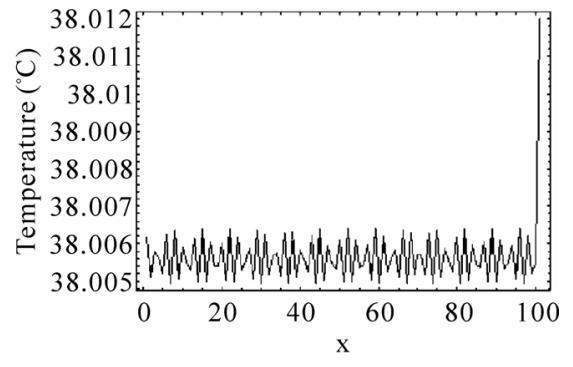

(b)

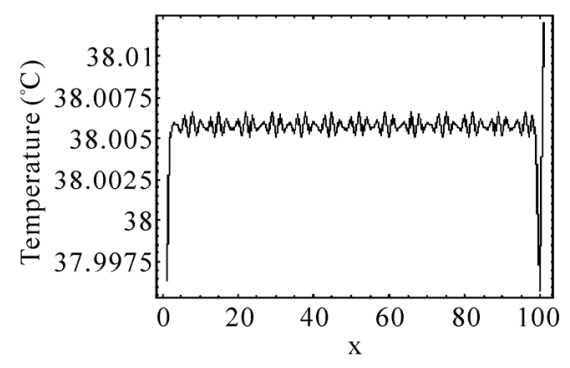

(e)

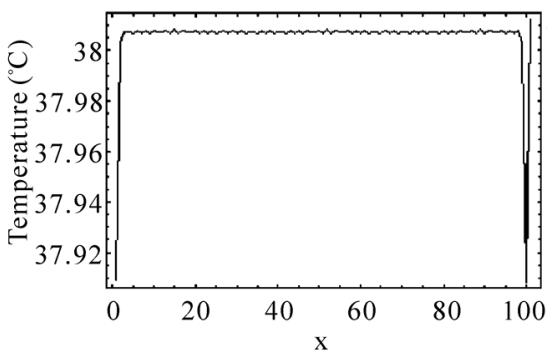

(h)

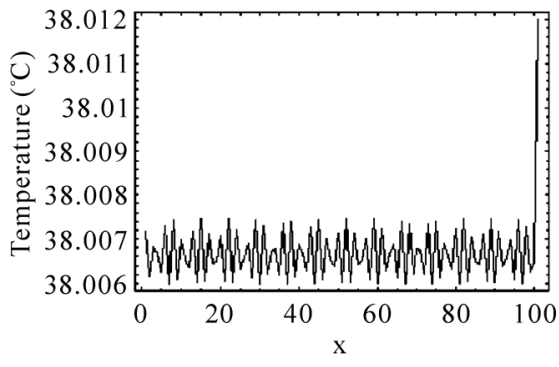

(c)

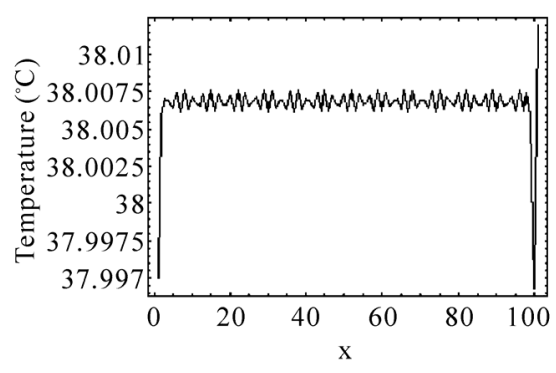

(f)

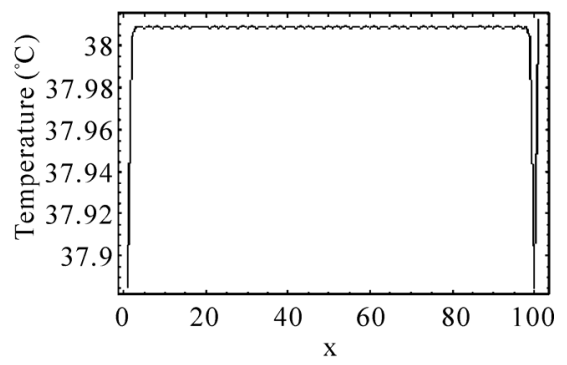

(i)

Figure 6. Temperature plotted against distance $x$ (in $\mathrm{mm}$ ) and at given time $t$ for $\omega_{0}=4.7619 \times 10^{-4} \mathrm{~s}^{-1}$ and $\omega_{1}=6.449 \times 10^{-3} \mathrm{~s}^{-1}$. Each row is for a different thermal diffusivity $\alpha: \quad \alpha=1.26311 \times 10^{-8} \mathrm{~W} / \mathrm{J} . \mathrm{m}^{2}$ for plots (a), (b), and (c), $\alpha=1.26311 \times 10^{-4} \mathrm{~W} / \mathrm{J} . \mathrm{m}^{2}$ for plots (d), (e), and (f), and $\alpha=1.26311 \times 10^{-2} \mathrm{~W} / \mathrm{J} \mathrm{m}^{2}$ for plots (g), (h), and (i), while each column is for different time: $t=50 \mathrm{~s}$ for plots (a), (d), and (g), $t=80 \mathrm{~s}$, for plots (b), (e), and (h), and $t=100 \mathrm{~s}$ for plots (c), (f), and (i). 


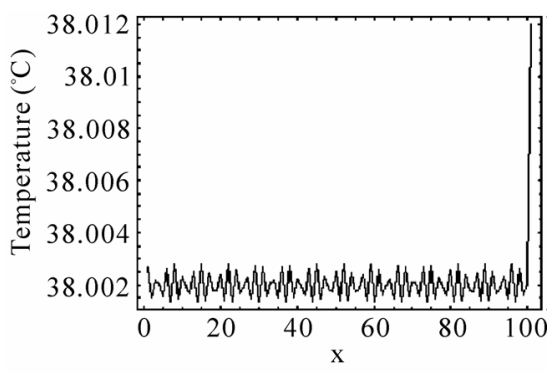

(a)

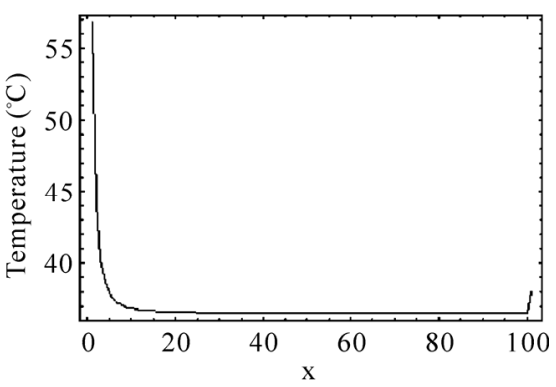

(d)

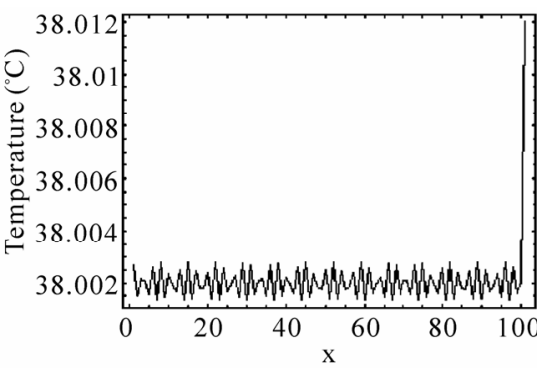

(b)

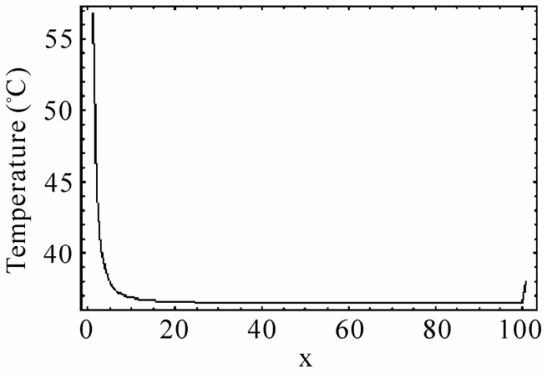

(e)

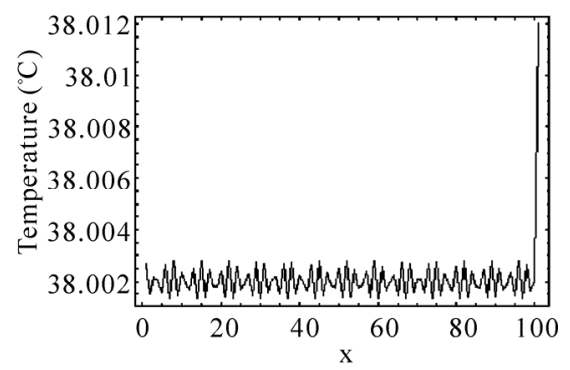

(c)

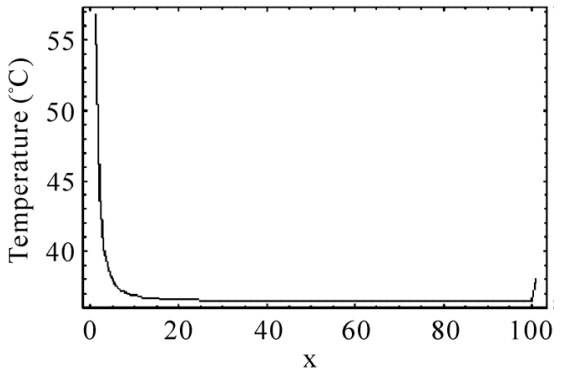

(f)

Figure 7. Plot of the temperature response along the distance $x$ (in $\mathrm{mm}$ ) from the heated surface at time $t=100 \mathrm{~s}$ for $\alpha=1.26311 \times 10^{-7} \mathrm{~W} / \mathrm{J} . \mathrm{m}^{2}$ and $\omega_{1}=\omega_{1}=6.449 \times 10^{-4} \mathrm{~s}^{-1}$ and for different values of temperature-independent perfusion component $\omega_{0}: \omega_{0}=4.7619 \times 10^{-4} \mathrm{~s}^{-1}$ for plots (a) and (d), $\omega_{0}=4.7619 \times 10^{-3} \mathrm{~s}$ for plots (b) and (e), and $\omega_{0}=4.7619 \times 10^{-2} \mathrm{~s}^{-1}$ for plots (c) and (f).

$\omega_{1}=\omega_{1}=6.449 \times 10^{-4} \mathrm{~s}^{-1}$. These plots show the temperature response along the distance $x$ from the heated surface at time $t=100 \mathrm{~s}$. For plots (a), (b), and (c), we have used the input temperature of arterial blood (28), while the input temperature of arterial blood (29) is used for plots (d), (e), and (f). Plots (a) and (d), (b) and (e), and (c) and (f) are obtained with $\omega_{0}=4.7619 \times 10^{-4} \mathrm{~s}^{-1}$, $\omega_{0}=4.7619 \times 10^{-3} \mathrm{~s}^{-1}$ and $\omega_{0}=4.7619 \times 10^{-2} \mathrm{~s}^{-1}$. From the plots of Figure 7 we can conclude that the effect of the temperature-independent perfusion component $\omega_{0}$ on the temperature response can be neglected.

\subsection{Effect of the Temperature-Dependent Perfusion Component $\omega_{1}$}

One of the effects of the temperature-dependent perfusion component $\omega_{1}$ can be obtained from Figure 8 where we plotted the temperature response as function of the distance $x$ from the heated surface at time $t=100 \mathrm{~s}$ and for $\alpha=1.26311 \times 10^{-7} \mathrm{~W} / \mathrm{J} \mathrm{m}^{2}$ and $\omega_{0}=4.7619 \times 10^{-4}$ $\mathrm{s}^{-1}$ For plots (a), (b), and (c), we used the input temperature of arterial blood (28), while plots (d), (e), and (f) are obtained with the use of the input temperature of arterial blood (29). Plots (a) and (d), (b) and (e), and (c) and (f) are obtained with $\omega_{1}=4.7619 \times 10^{-4} \mathrm{~s}^{-1}$, $\omega_{1}=4.7619 \times 10^{-3} \mathrm{~s}^{-1}$ and $\omega_{1}=4.7619 \times 10^{-2} \mathrm{~s}^{-1}$. It follows from the plots of Figure 8 that the temperature response increases with the temperature-dependent perfusion component $\omega_{1}$.

\section{CONCLUSION}

In this paper, the one-dimensional steady-state bioheat transfer model of the living tissues has been taken into account based on the modified (nonlinear) Pennes equation, and the corresponding equation has been solved both analytically and numerically. The analytic solution expressed by the Jacobi elliptic functions, trigonometric functions and rational functions are derived to obtain the temperature changes with the variation of the distance from the heated surface. The analytic solutions have been used to investigate the effects of the thermal diffusivity, the temperature-independent perfusion component, and the temperature-dependent perfusion component on the temperature distribution. The obtained analytic solution can provide a good knowledge of thermal behavior of living tissues, which are valuable for the measurement of thermal parameters, the reconstruction of the temperature field and the thermal diagnosis and treatment, the dosimetry, and the human implantation smart devices. 


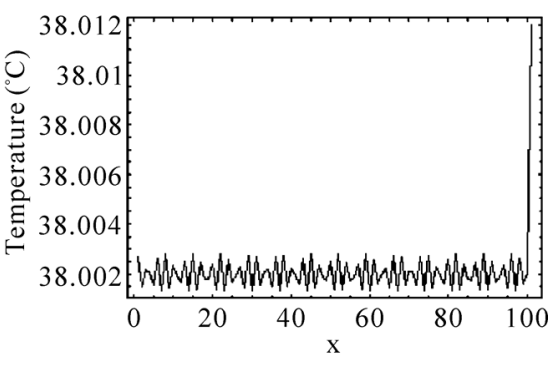

(a)

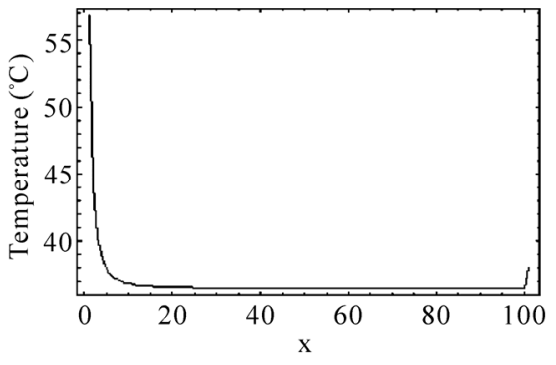

(d)

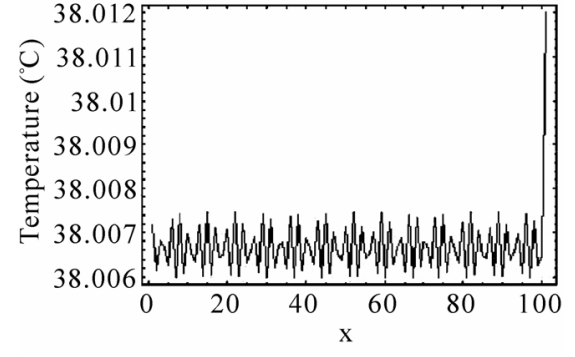

(b)

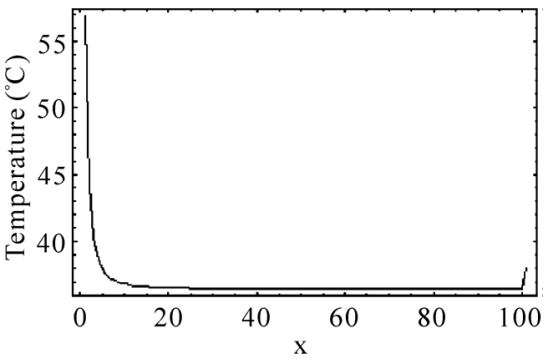

(e)

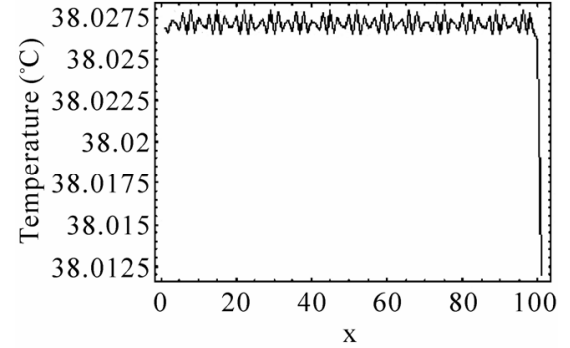

(c)

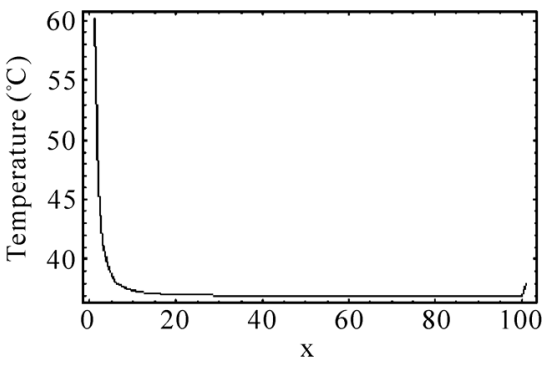

(f)

Figure 8. Temperature response along the distance $x$ (in $\mathrm{mm}$ ) from the heated surface at time $t=100 \mathrm{~s}$ for $\alpha=1.26311 \times 10^{-7} \mathrm{~W} / \mathrm{J} . \mathrm{m}^{2}$ and $\omega_{0}=4.7619 \times 10^{-4} \mathrm{~s}^{-1}$ and for different values of $\omega_{1}: \omega_{1}=6.449 \times 10^{-4} \mathrm{~s}^{-1}$ for plots (a) and (d), $\omega_{1}=6.449 \times 10^{-3} \mathrm{~s}^{-1}$ for plots (b) and (e), and $\omega_{1}=6.449 \times 10^{-2} \mathrm{~s}^{-1}$ for plots (c) and (f).

\section{ACKNOWLEDGEMENTS}

The authors thank NSERC for their financial support, CMC Microsystems and LIMA "Laboratoire d'Ingénierie des Microsystèmes Avancés” at UQO (Université du Québec en Outaouais) for providing design tools, support and associated technologies.

\section{REFERENCES}

[1] Chato, J.C. (1981) Reflection on the history of heat and mass transfer in bioengineering. Journal of Biomechanical Engineering, 103, 97-101.

[2] Pennes, H.H. (1948) Analysis of tissue and arterial temperatures in the resting human forearm. Journal of Applied Physiology, 1, 93-122.

[3] Davalosa R.V. and Rubinsky B. (2008) Temperature considerations during irreversible electroporation. International Journal of Heat and Mass Transfer, 51, 56175622.

[4] Niu J.-H., Wang H.-Z., Zhang H.-X., Yan J.-Y. and Zhu, Y.-S. (2001) Cellular neural network analysis for two dimensional bioheat transfer equation. Medical \& Biological Engeering Computing, 39, 601-604.

[5] Ginter, S. (2000) Numerical simulation of ultrasoundthermotherapy combining nonlinear wave propagation with broadband soft-tissue absorption. Ultrasonics, 37, 693-696.

[6] Lang J., Erdmann, B. and Seebass, M. (1999) Impact of Nonlinear Heat Transfer on Temperature Control in Regional Hyperthermia. IEEE Transactions on Biomedical Engineering, 46, 1129-1138.

[7] Arkin, H., Xu, L.X. and Holmes, K.R. (1994) Recent developments in modeling heat transfer in blood perfused tissues. IEEE Transactions on Biomedical Engineering, 41, 97-107.

[8] Alekseev, S.I. and Ziskin, M.C. (2009) Influence of blood flowand millimeter wave exposure on skin temperature in different thermal models. Bioelectromagnetics, 30, 52-58.

[9] Nyborg, W.L. (1988) Solutions of the bio-heat transfer equation. Physics in Medicine and Biology, 33, 785-792.

[10] Shih, T.-C., Yuan, P., Lin, W.-L. and Kou, H.-S. (2007) Analytical analysis of the Pennes bioheat transfer equation with sinusoidal heat flux condition on skin surface. Medical Engineering \& Physics, 29, 946-953.

[11] Bodo, E., Jens, L. and Martin, S. (1998) Optimization of temperature distributions for regional hyperthermia based on a nonlinear heat transfer model. Annals of the New York Academy of Sciences, 858, 36-46.

[12] Seese, T.M., Harasak, H., Saidel, G.M. and Davies, C.R. (1998) Characterization of tissue morphology, angiogenesis, and temperature in the adaptive response of muscle tissue to chronic heating. Journal of Technical Methods and Pathology, 78, 1553-1562.

[13] Davies, C.R., Saidel, G.M. and Harasaki, H. (1997) Sensitivity analysis of 1-D heat transfer in tissue with temperature-dependent perfusion, Journal of Biomechemical. Engeeering, 119, 77-80.

[14] Kengne, E. and Liu, W.M. (2006) Exact solutions of the derivative nonlinear Schrödinger equation for a nonlinear transmission line, Physical Review E, 73, 1-8.

[15] Lakhssassi, A., Kengne, E. and Semmaoui, H. (2010) Investigation of nonlinear temperature distribution in biological tissues by using bioheat transfer equation of 
Pennes'type. Journal of Natural Sciences, 2, 131-138.

[16] Kengne, E. and Vaillancourt, R. (2009) Exact equilibrium solutions of a diffusion equation with a nonlinear diffusion term by means of Jacobian elliptic functions. Integral Transforms and Special Functions, 1, 1-18.

[17] Weierstrass K. and Mathematische W.V. (1915) Johnson, New York, 4-16.

[18] Whittaker, E.T. and Watson, G.N. (1927) A Course of Modern Analysis, Cambridge University Press, Cambridge.

[19] Chandrasekharan, K. (1985) Elliptic Functions. Springer, Berlin.

[20] Schürmann, H.W. and Serov, S.V. (2004) Traveling wave solutions of a generalized modified. Kadomtsev-Petviashvili equation, Journal of Mathematical Physcis, 45, 2181-2187.

[21] Abramowitz, M. and Stegun, A.I. (1972) Handbook of
Mathematical Functions. 9th edition, Dover Publications, New York.

[22] Byrd, P.F. and Friedman, M.D. (1971) Handbook of elliptic integrals for engineers and scientists. 2nd Edition, Springer-Verlag, Berlin.

[23] Schürmann, H.W., Serov, V.S. and Nickel, J. (2006) Superposition in nonlinear wave and evolution equations. International Journal of Theoretical Physics, 45, 10571073.

[24] Kou, H.S., Shih, T.C. and Lin, W.L. (2003) Effect of the directional blood flow on thermal dose distribution during thermal therapy: an application of a Green's function based on the porous model. Physics in Medicine and Biology, 48, 1577-1589.

[25] Press, W.H., Flannery, B.P., Teukolsky, S.A. and Vallerling, W.T. (1992) Numerical Recipes in Fortran. Cambridge University Press, Cambridge. 